\title{
Behavioral therapy for temporomandibular disorders
}

\author{
Richard Ohrbach ${ }^{1}$, Sonia Sharma ${ }^{1,2}$ \\ ${ }^{1}$ Department of Oral Diagnostic Sciences, University at Buffalo School of Dental Medicine, Buffalo, NY, USA; ${ }^{2}$ Department of Orofacial Pain and \\ Jaw Function, Faculty of Odontology, Malmö University, Malmö, Sweden \\ Contributions: (I) Conception and design: Both authors; (II) Administrative support: None; (III) Provision of study materials or patients: None; \\ (IV) Collection and assembly of data: None; (V) Data analysis and interpretation: Both authors; (VI) Manuscript writing: Both authors; (VII) Final \\ approval of manuscript: Both authors. \\ Correspondence to: Richard Ohrbach. Department of Oral Diagnostic Sciences, 355 Squire, University at Buffalo School of Dental Medicine, Buffalo, \\ NY 14214, USA. Email: ohrbach@buffalo.edu.
}

\begin{abstract}
Behavior-what a person does to attain a goal—relevant to temporomandibular disorders (TMDs) can be classified into three types: organ system-level functional behaviors, organ system-level non-functional behaviors, and person-level behaviors. Despite decades of productive research regarding the importance of behavior, taken broadly, as encompassing multiple risk factors for another prevalent musculoskeletal pain disorder (low back pain), behavioral research related to TMDs remains in the early stages. Clinically, behavioral factors are complex with regard to adequate assessment, and they require specific conceptual and management skills. Consequently, providing due diligence to their importance is challenging in many medical arenas. The sparse data that exist supporting the role of behavior in TMD onset and persistence indicate that excessive extent of masticatory system non-functional and possibly functional behaviors contributes to painful TMD onset and appear to contribute to chronicity. In addition, TMD-relevant behaviors can be amplified by chronic pain, among other stressors, suggesting a complex reciprocal relationship. Both the reciprocal relationship between TMD pain and functional and non-functional behaviors and the person-level behaviors create multiple interactive feedback loops which then serve as barriers to behavioral change. These barriers need to be addressed in a step-like manner with treatment, such that motor control and sensory perception undergo re-learning. At present, the evidence regarding treatments or their efficacy is minimal and mostly indirect. The field needs to develop better theories regarding how behavior fits within the available evidence pertaining to TMD etiology and persistence. With better theories and transfer of knowledge from other pain fields, better treatment research can be implemented for TMDs.
\end{abstract}

Keywords: Temporomandibular disorders (TMDs); behavior; pain; assessment; management

Received: 02 September 2020; Accepted: 15 May 2021; Published: 10 December 2021.

doi: $10.21037 /$ fomm-20-65

View this article at: http://dx.doi.org/10.21037/fomm-20-65

\section{Overview}

Behavior can be a deceptively complex concept. At its simplest, behavior-the final result of a coordinated set of motor unit activations in the muscles necessary for the particular movement-is what organisms do in order to achieve a goal. For present purposes, behaviors can be separated into three types: organ system-level functional behaviors, organ system-level non-functional behaviors, and person-level behaviors. If the instrumental purpose is to communicate, show dismay, or meet nutritional needs, then the simple masticatory system functional behaviors might be to speak, frown, or chew, respectively. But "simple" can be elusive: all 3 behaviors are far more complex than they appear. For example, speech and facial expression are highly nuanced in their goal of maintaining social standing simultaneous with obtaining needs from others. Similarly, chewing is influenced by the need to maintain social eating etiquettes, but chewing requires a complex motor pattern in terms of coordinating eight primary muscles 
of mastication and multiple accessory muscles; adjusting trajectories, force, and timing; and integrating constantly changing sensory feedback-all the while avoiding biting on the tongue in lieu of the food bolus (1). While each of the above behaviors has a specific and essential purpose, the complexity underlying their performance also means that they are also influenced by multiple determinants (for example, emotional states) which can lead to variation in performance; those variations can become clinically relevant. Non-functional behaviors involving the masticatory system, usually termed parafunctional or overuse behaviors, reflect coping responses or habit patterns without instrumental purpose. Behavior also encompasses more patterns at the person-level, which are oriented towards addressing higher-level needs. Clinical problems, such as temporomandibular disorders (TMDs) a group of musculoskeletal disorders affecting the masticatory system, involve all three types of behaviors.

"Behavioral therapy for TMDs" (our title) accurately conveys the eventual goal of this paper yet the first term requires in all instances careful description. Our purpose here is to explore the current status of behavior and its clinical implications regarding pain in general and TMDs in particular. Given the integrated character of behavior (e.g., motor unit activation coupled with role of joint afferents in regulating the motor unit activation), behavior therapy need not distinguish extra-articular TMDs from intra-articular TMDs. Rather, the focus should be on the problematic behaviors that inherently involve both joint and muscle. Finally, because the role of behavior in TMDs is an area that is currently in great flux, strong evidence is sparse. An effective systematic review is precluded at this time. Consequently, we turn to an integrated perspective based on how the TMD field has developed with regard to behavioral concerns, the dynamics and complexity of behavior (so that clinical assessment can be enriched), and assessment and management perspectives relevant to all 3 types of behaviors.

\section{Background, or why we know so little about behavior and TMDs}

The potential importance of behavior to the management of TMDs has been recognized since the 1950s $(2,3)$. While some of these early views were very insightful and current in today's term, most primarily focused on only masticatory system parafunctional behaviors and not behavior more broadly. Moreover, the primary parafunctional behaviors of interest were restricted to clenching or grinding of the teeth (collectively regarded as bruxism). While the early views sometimes distinguished behaviors that occurred during waking $v s$. sleeping states, more often the distinction was ignored. Subsequent knowledge strongly suggests that these two states should be distinguished for assessment, classification, and management, and clenching and grinding represent only two behaviors among many waking parafunctional behaviors of the masticatory system (4-6). Moreover, distinguishing clenching from grinding during sleep is probably less important than their temporal association with pain $(7,8)$. Consequently, from the 1950 s through the 1980s, the considerable clinical interest and investigation into these behaviors and their relation to TMDs was highly limited: the focus was primarily on sleep bruxism, not waking behaviors; operational definitions for either bruxism or TMD were seldom rigorous, if employed at all; and a causal relationship of sleep bruxism to TMD pain was largely accepted as self-evident based on crosssectional observational data. A belief, however, persisted among many clinicians and investigators that waking behaviors were an important contributor to TMDs, and investigators were increasingly expressing doubt regarding the causal role of sleep bruxism for TMDs. The absence of a conceptual or taxonomic framework for these waking behaviors and for their measurement resulted in a wide range of perspectives, and knowledge development was not systematic. Back-pain research during this same period, in contrast, incorporated behavioral concepts extensively (9-12). Much of what we might describe in this paper as "new" for TMDs was previously identified for back pain.

In recognition that a formal descriptive taxonomic system was needed for TMDs in order to foster far better research, the Research Diagnostic Criteria for TMDs (RDC/TMD) were developed based on core design principles $(13,14)$ leading to physical diagnoses (Axis I) that captured the most prevalent TMDs and to assessment of the person with pain (Axis II) that captured the most common comorbid characteristics known at that time. The developers of the RDC/TMD acknowledged that overuse behavior such as bruxism was probably important for TMDs, based on the existing data, yet assessment and classification of bruxism was not included in the RDC/TMD because evidence for reliable assessment did not exist. Consequently, neither bruxism nor any other type of oral parafunctional behavior was formally assessed within the RDC/TMD except for two questions in the Patient History Questionnaire: "Have you been told, or do you notice that you grind your teeth or clench 
your jaw while sleeping at night?" and "During the day, do you grind your teeth or clench your jaw?" During this period, research regarding sleep bruxism and its relationship to pain increased greatly (15-17), whereas research regarding waking behaviors associated with the masticatory system continued to lag, with some notable exceptions $(18,19)$.

Approximately 10 years following publication of the RDC/TMD, the reliability and validity of the physical diagnoses within the RDC/TMD was formally tested in a large multi-site study (20). The project investigators were further tasked with potential expansion of the Axis II assessment protocol, which included better assessment of behavior associated with the TMDs (21). By the early 2000s, chronic pain research, including behavioral aspects of chronic back pain, had led to convincing evidence that behavior can both contribute to, and be exacerbated by, chronic pain (22-30). Yet, parallel knowledge regarding waking behavior and TMDs was notably absent despite, if anything, an increased sensitivity by clinicians that such behaviors were important; management to that date in practice settings was primarily implemented via principles initially advocated in the 1950s (for example, the single directive to the patient: "lips together, teeth apart") (2), while management in university settings often incorporated some form of electromyographic (EMG) biofeedback as a direct intervention into waking parafunctional behavior (31-34). There was little integration between these approaches.

While the chronic pain literature was replete with evidence supporting both system-specific behavioral problems (such as guarding behaviors to protect against pain -in the case of low back pain) as well as more general illness-associated behaviors (such as avoidance of responsibilities, excessive rest, and pain-related disability), this aspect of behavior was typically not integrated into the realm of TMDs. Instead, TMDs were regarded as uniquely a function of structural aberrations and different in kind from all other chronic musculoskeletal pains. A specific clinical focus on behaviors that putatively affected the masticatory system was largely excluded, and as pain research revealed increasing links between chronic muscle pain and the biopsychosocial model, a belief emerged in TMD treatment settings that intra-articular disorders were "different" and that the biopsychosocial model was therefore not applicable to such disorders. Certainly, consistent evidence has demonstrated that individuals with only intra-articular TMJ problems have less psychological distress compared to those with only myofascial pain disorders $(35,36)$. Yet, absence of distress does not mean that behavioral factors affecting the physical condition are absent. The field of medicine had clearly recognized that a disorder as structural as knee osteoarthritis exhibited pain more responsive to mood states than to degree of diseaserelated tissue damage, and behavior was a primary factor in determining both disease and function outcomes $(37,38)$.

In summary, the field of TMDs has been largely isolated from chronic musculoskeletal disorders in general, resulting in notable differences within the TMD field: an increased emphasis on identifying structural changes as risk factors, a primary treatment focus on structural factors (whether deemed a risk factor or not), and neglect of behavioral factors and central dysregulation now known to be associated with chronic pain disorders (14). Developments in waking behavior and its clinical implications regarding pain generally and TMDs in particular will be further explored. We will not explore the role of sleep bruxism, a behavior of a different type occurring in a different state of consciousness and perhaps best classified as a movement disorder; for that, the reader is directed to other sources (39-41).

\section{A pathogenetic view of behavior and TMD}

Functional behaviors often become automatic after sufficient learning such that when performed in a customary context by a healthy individual, the behavior becomes easy to perform and occurs without conscious planning for how the behavior should unfold. In such circumstances, we forget about behavior as an important concept because it is seemingly an invisible bridge from one state (e.g., I am hungry) to the next state (e.g., I am satisfied). In this circumstance, the end goal of the behavior is to maximize the probability of experiencing the perception associated with the desired goal. In response to various circumstances, the goal of a behavior may shift, however, from maximizing a desired enjoyable (hedonic) perception to avoiding an aversive perception. Rather than appetitive, behavior becomes protective and, consequently, becomes much more complex in both its execution (with respect to how the behavior is implemented by the motor cortex) and its intention. Negative reinforcement replaces positive reinforcement.

Clinical problems such as TMDs can result from more of a behavior, characterized as increased frequency, duration, or magnitude of the behavior. Both functional and nonfunctional behaviors are important. Functional behaviors considered part of normal jaw use, such as chewing or speaking, can be taken to excess but whether they, in excess, 
are primary causes of clinical problems or whether they are aggravating factors once a disorder is present is not yet known. In contrast, sudden excessive use of chewing gum can easily lead to localized muscle spasm that can readily resolve by immediately terminating the excessive gum chewing. Other functional but perhaps non-normative behaviors involve bracing the jaw such as playing violin or holding a telephone handset between shoulders and mandible and these behaviors, if done to excess, are believed to be capable of causing musculoskeletal problems (42).

Multiple types of non-functional (overuse) behaviors are possible such as clenching, tooth tapping, tooth pressing, tongue pushing, tongue sucking, lip biting, jaw bracing, and jaw tension. Increases in the behavior(s) are thought to be particularly important risk factors for TMDs (43-49). The mechanism underlying how non-functional behaviors might lead to the development or aggravation of pain or dysfunction remains poorly understood. Associations demonstrated in cross-sectional studies (50-52) have been the most frequent type of study from which interpretations of causation have been inappropriately made. A small number of well-performed studies, primarily through ecological monitoring of behavior and time-locked symptom changes, provide more convincing evidence $(43,53)$.

Clinical problems can also result from alterations in how the behavior is executed. Perhaps the best known example is musicians who overuse certain fine muscles of the hands leading to fatigue and unconscious compensatory development of new motor unit recruitment patterns that allow the individual to continue to play, which then increases the risk for development of tendon injuries that can completely stop all performance (42). The muscles of mastication are equally susceptible to such problems. For example, the presence of odontogenic pain will alter chewing pattern, typically one that is asymmetrical, in order to avoid loading of the involved tooth; unilateral chewing increases risk for developing a TMD (54). In this instance a functional behavior becomes abnormal in its execution, which is both unconscious (e.g., pain is a stunningly effective behavioral change agent) and conscious (e.g., avoidance of pain is also a stunningly effective behavioral change agent).

A unidirectional path-from excessive behavior to pain onset, aggravation, or both-appears to be an incomplete description of behavior and its relationship to clinical musculoskeletal disorders. The available evidence indicates that clinical problems such as TMDs can also result in increased frequency, duration, and magnitude of a behavior as well as result in alteration of behavior execution as attempts to adapt to any pain or limitation in movement (55). We previously reported a very large magnitude of association $(\mathrm{OR}=16.8,95 \% \mathrm{CI}$ : 8.6-32.9) between a global index of oral overuse behaviors and chronic painful TMD, in contrast to the magnitude of association with other factors associated with painful TMD—for example, TMJ sounds on palpation during movement $(\mathrm{OR}=2.6,95 \%$ CI: 1.9-3.7) or female sex (OR $=4.0,95 \%$ CI: 2.6-6.0) $(50,56)$. This very large association between parafunctional behaviors and chronic painful TMD in a cross-sectional study design was interpreted as likely reflecting a reciprocal process: effects of the behaviors on TMD chronicity as well as the behaviors being respondent to pain, other mechanical jaw problems, or other more general factors such as stress.

Behavior also refers to more global patterns of action by the individual, such as operant behavior-a specific behavioral pattern by the individual to control how one's needs are met by another person; illness behavior-the act of being ill, seeking care, taking medication; or fearavoidance behavior-localized guarding coupled with withdrawal from normal use of the affected body regionitself a behavior characterized by, paradoxically, inaction. These types of patterns can (and probably most often do) exist in addition to local patterns, described above. Consequently, the entire performance of chewing that also includes congruent facial expressions could be used as a means to control another through chewing-related pain behaviors; or chewing could be slow in a way in which an individual demonstrates to others that illness is present; or chewing could become highly limited due to fear-avoidance beliefs resulting eventually in progressive atrophy of the masticatory muscles. In each of these three instances, the behavior can be quantified in terms of frequency, duration, and force; and in each instance, the intention behind the behavior can be identified. In addition, each of these three instances could occur in the same individual within a short period of time. Understanding the local behaviorchewing-requires understanding the individual; the neurophysiology alone underlying the different ways in which chewing might manifest as a behavior would be insufficient for understanding how a behavior, such as chewing, might be a problem.

Behavior is the final output of motor activation, and yet that same behavior is also a source of input to the nervous system: the execution of a given behavior serves as the starting point of a new cycle of central nervous system activity wherein the outcome of that behavior, measured 
as both sensory experience of the behavior as well as the instrumental goal of the behavior, becomes the input for a new cycle of activity. While a given behavior can be readily classified by the observer based on its beginning and ending, behavior for the individual is a continuous stream, that is, behavior is inherently dynamic and represents simultaneously the outcome of controlled motor activation as well as the sensory and motivational state of the organism as it exists at that moment. For patients whose clinical condition is linked to behavior, both levels are important. Both levels, in turn, contribute to the reciprocal process identified above.

Behavior, in relation to a disorder such as TMDs, can therefore be considered from two levels: the behavior as a discrete form of activity, and behavior as the sum total of all action by the individual on the world around themwhat may be termed organismic behavior. Discrete behavior performed in an excessive manner (to be defined) - that is, beyond normal functional requirements-is generally assumed to be pathogenic towards local tissue. Organismic behavior, in contrast, acts on the individual as a whole: withdrawal behavior leads to depression, for example. A given behavior can exist at both levels simultaneously: protecting the back from excessive movement, called guarding behavior, entails hyperactivity of the associated muscles in one part of the back (and is typically asymmetrical), and hyperactivity can lead to fatigue, incoordination, muscle contracture, and pain; guarding behavior also limits activity engagement and thereby decreases pleasure and social support and contributes to depression. Depression leads to further inactivity and guarding. Clearly, behavioral problems must be identified and physical treatments for TMDs must be aligned with restoration of adaptive behavior (57).

A multivariable model examining contributing factors to pain onset and pain chronicity highlights both unique pathways as well as likely recursive pathways between risk factors and the transition to chronicity and maintenance of chronicity (58). Based on the mixture of biopsychosocial variables contributing to pain progression, behavior encompasses many of those processes. However, we have little direct evidence regarding this proposed reciprocal pathway or, by extension, the proposed dynamic process whereby both primary and reciprocal patterns augment each other in terms of possible behavioral persistence and symptom aggravation. An attempt to depict this complexity for clinicians is notable for its description of how the clinician must understand and actively manage the multiple feedback processes inherent in these patterns; the therapeutic task is clearly one of reteaching normal movement and posture to the patient (59). The very frequent incomplete response to simple behavioral therapies for non-functional behaviors (e.g., "just stop doing the parafunctional behavior") (60-62) may point to the potential importance of more complex mechanistic pathways such as this proposed reciprocal one as well as the necessity to integrate, in one's treatment approach, problems in functional behaviors (e.g., altered chewing) as well as problems in person-level behaviors (e.g., fear-avoidance). Consequently, we suggest that possible bi-directional and multi-level causal links could be present in individuals with chronic TMDs. The therapeutic challenge extends well beyond the available evidence from clinical trials.

\section{Masticatory system functional behaviors}

\section{Assessment}

Behaviors considered to represent instrumental jaw use include chewing, opening the mouth, verbal and non-verbal expression, and others such as musical instrument playing. Determining normal $v s$. abnormal extent of these behaviors is very difficult due to the extreme extent each of these can occur within individuals who are (and remain) completely asymptomatic. Consequently, the useful clinical markers for abnormality appear to be alteration in how the behavior is executed and frequency.

Alterations in behavioral execution occur with chewing (such as unilateral chewing in response to a symptomatic tooth), mandibular movement (such as deflected opening in response to disc clicking or unilaterally shortened muscle), speech (such as continuing to speak when masticatory muscles are fatigued), and musical instrument performance (such as with small string, wind, or brass instruments). For wind and brass instruments, alterations are typically evident in strained embouchure which is best determined by the individual's music teacher and managed accordingly. Alterations in execution can also occur as a result of very hurried chewing, for example, and pushing the central pattern generator for mastication beyond coordinated movement. High frequency of usage typically occurs with speech, bracing the mandible for musical instrument positioning, and rotating the head and mandible to hold a phone (or any object) between mandible and shoulder. Alterations in execution as well as high frequency beyond interoceptive signals indicative of strain are assumed to 
include recruitment of less-optimal motor units in order to continue to function, which can lead to dysfunctional recruitment and further fatigue.

In all instances, the patient history will indicate a change in the behavior, comparing pre-symptom state to the present symptom state. The clinician appraisal is therefore based on relative changes in execution, as inferred from the history, and relative changes in frequency of the putatively problematic behaviors, coupled with examination findings of alterations in movement. For example, chewing could have always been bilaterally symmetrical prior to the breakage of a tooth, after which unilateral chewing began to avoid the tooth; notably, after the tooth was repaired the unilateral chewing persisted-simply because new learning led to implementation of a new automatic (habitual) pattern.

\section{Management}

Restoration of normal behavioral pattern and expectations is the therapeutic goal for problematic functional behaviors (63). Little evidence exists for the below principles in specifically managing the masticatory system in relation to a TMD; instead we rely upon general principles established elsewhere (64-67). Reinstating bilateral chewing is accomplished via conscious retraining by the patient; a 2-week period could be used during which softer foods are consumed in order to facilitate the change from unilateral to bilateral chewing, and then gradually returning to normal textured foods. Bilateral chewing includes alternating side of the bolus, splitting the bolus to chew both sides simultaneously, or any combination. The training period in using soft diet should be time-limited; excessive use of soft diet contributes to further problems, while rapid return to normal textured food diet facilitates symptoms improvement (68).

Pioneering research (69) regarding altered mandibular opening movement pattern in the frontal plane as well as acceleration and deceleration during the openclose movements in response to disc displacement with reduction has led to the assumption that such patterns are an intrinsic consequence of the mechanics of an internal derangement. An alternative interpretation, such as from the fear-avoidance model $(27,70,71)$, suggests that altered opening patterns emerge due to unconsciously avoiding the difficulty endangered by an internal derangement, and that over time the compensatory pattern becomes the dominant (habitual) one and, by extension, becomes the new normal within which joint function (and joint proprioception) accommodates. Such patterns also occur in response to musculoskeletal pain (72). Correcting deviations in the frontal plane as well as changes in speed of movement are best addressed with visual feedback: a mirror for the patient to observe the movement, directions from the clinician regarding what is normal, and gradual retraining of the movement in order to return to a more normal pattern. Such strategies represent emerging understanding of altered body representation and movement patterns as treatment targets for musculoskeletal disorders (73-76).

Highly frequent speech, such as by teachers or individuals in the service industry, can become an aggravating factor for overuse; teaching use of intermittent relaxation during non-speech moments can allow for recovery rather than the recurrent strain that persistent overuse of speech can lead to, given the presence of a musculoskeletal disorder.

\section{Masticatory system non-functional behaviors}

\section{Assessment}

Traditionally, waking parafunctional behaviors have been assessed by interview or self-reported questionnaire regarding presence or absence of clenching or grinding behaviors associated with the teeth. The behaviors were considered to be dichotomous-absent (normal) or present (abnormal) - with regard to a potential contribution to a TMD. An early landmark publication of individuals from 15-65 years of age provided a life-course perspective regarding a more complex occurrence of these behaviors: tooth clenching was reported by $42 \%$ of individuals during $35-44$ years of age, and as low as 15\% during other ages; soft tissue biting was reported by 19\% during 15-24 years of age, and then decreasing to $10 \%$ for the remaining ages; object biting was reported by $23 \%$ during $15-24$ years of age, and thereafter only a few percent for the remaining age groups; and tongue pressing was reported by $10 \%$ across all age groups (77). The results of that investigation indicated that multiple types of parafunctional behaviors exist, that positive response rates were increased when various behaviors were queried rather than only clench or grind, and that some behaviors have a lifetime pattern whereas other behaviors are life stage-specific. Similar findings of multiple behaviors have been observed, for example, in nursing students (78); at least daily, 39\% reported touching or holding teeth together, $15 \%$ holding jaw rigid, and 24\% biting objects.

An important implication of those findings is that since waking parafunctional behaviors are more often 
unconsciously rather than consciously performed, a single question of "clench or grind?" does not sufficiently probe memory, and without a sufficient probe, false negatives are probable. A series of questions prompts respondents to more carefully consider each behavior, often augmented by testing the behavior and directly probing memory of proprioceptive experience. Ecological momentary assessment data provide consistent estimates. When prompted in the field by a pager to self-monitor (79), nonTMD controls reported their teeth were in contact $45 \%$ of the time, while individuals with either disk displacement or myofascial pain reported $56 \%$ of the time, and individuals with both myofascial pain and TMJ arthralgia reported $73 \%$ of the time. When similarly prompted in the field by pocket computer (4), a mixture of individuals (TMD-free, or any type of TMD) reported varying proportions (range, 2-26\%) of 11 different behaviors per prompt, and reported any of 6 behaviors such as clench or press $76 \%$ of the time. Overall, under-assessment has been likely in both clinical settings and in published research, leading to underestimation of associations with TMDs.

Collectively, these findings point to a spectrum of types of behaviors and number of different behaviors one individual might do, varied intensity and varied frequency of different behaviors across individuals, and a trait-like aspect for certain behaviors that are lifelong. In addition, some individuals are far more prone to such behaviors. Being considered within a spectrum also suggests that "parafunction" as a classification may miss another important perspective: these behaviors lie within a continuum that is dependent on extent (frequency, duration, and force magnitude) of each reported behavior, summed across all of the reported behaviors). A critical threshold likely exists in that continuum separating "normal" from clinically abnormal and potentially pathogenic. Just as everyone exhibits at least a small extent of sleep bruxism some of the time $(80,81)$, nearly everyone engages in some type of waking parafunctional or overuse oral behavior, at least some of the time. The variability in types of behaviors as well as their frequency is probably influenced by multiple mechanisms: the behaviors can occur both unconsciously as an autonomous habit, unconsciously as reactive to other events such as back pain or physical strain (e.g., weight training), unconsciously as adaptive behaviors, and consciously (or unconsciously) as a coping mechanism to other events such as stress or anger.

Among possible approaches for simple routine assessment of these behaviors, the self-reported Oral
Behaviors Checklist $(5,6)$ has perhaps the best evidence for reliability and validity and was consequently adopted as a standard instrument within Axis II of the DC/TMD (82). The 21 items probe sleep bruxism and posture (2 items), non-functional behaviors such as clenching or jaw bracing (11 items), and functional behaviors such as playing a musical instrument, unilateral chewing, and sustained talking (8 items). The instrument uses a 5-point rating scale from none of the time to all of the time. Semantically, the terms are well-understood (5), similar behaviors such as "clench" vs. "press" have distinct EMG signatures (83), and self-report assessment via a paper instrument accords very well with field monitoring of the behaviors (4). Whether all of these items, as indicators of different types of behavior, are equally important contributors to TMD onset or aggravation has not yet been determined. Consequently, the major scoring to date has utilized a sum score from all 21 items $(46,50)$ and best practice for clinical usage is to further interview the patient regarding positive responses in order to confirm frequency, explore context (i.e., behavioral antecedents, setting), and identify consequences. Patients may have very informed responses to each of these 3 interview questions, or these questions may point to as-yet unknown aspects of the person's behavior and suggest an action plan for daily monitoring and then reassess. Associations between OBC high scores and TMDs have been reported from many settings $(6,47,50)$; clearly, the extent of parafunctional behaviors exists along a continuum, and a low extent should be considered "normal" and not pathogenic.

Ultimately, a questionnaire such as the $\mathrm{OBC}$ is only the beginning of assessment; the patient must recognize the behaviors, their frequency, their context, and their consequences (e.g., pain episodes, increased temporomandibular joint clicking), but a full recognition can take months of engagement through treatment, so expectation of understanding by both patient and clinician at the early stages should be realistic. An appraisal based on the initial information (such as responses to the OBC and related interview) is required to determine if change in the behaviors should be a goal for treatment. The initial assessment must also lead to patient engagement; without that, behavioral management cannot succeed.

\section{Management}

When extent of non-functional behaviors is high, then treatment should focus on attainment of a neutral position of the mandible which is incompatible with any 
parafunctional or overuse behavior (with the important and paradoxical exception of muscle strain sometimes emerging in the goal to attain that neutral position). The goal is to retrain habitual and respondent parafunctional behaviors, replacing what is customary with a new habitual neutral position of the mandible. Defining neutral, however, is challenging; for some, "neutral" is the location of the mandible where EMG measure of the masticatory muscle activity is minimal (84), whereas for others "neutral" is defined interoceptively as absence of "tension". The former is typically achieved with tongue tip held in the floor of the mouth, while the latter is achieved with tongue tip placed at anterior palate (the so-called "n-position"), at least at the beginning $(84,85)$. Both approaches are useful, but lead to different positions of the mandible as measured in the vertical plane-with the mandible positioned more inferior in order to attain minimal EMG. The minimal EMG approach $v s$. a "relaxed, non-tense position" has been extensively researched with other muscles $(86,87)$, and while minimal EMG is certainly associated with the least perception of any tension, it is also perhaps unnecessary from what might be described as clinically "normal", correlates poorly with symptom reduction, and represents a therapeutic goal that may be more difficult relative to ecologically useful training.

There is little systematic evidence for efficacy of tongue posture training alone $(88,89)$, yet some form of relaxation is used frequently and with good efficacy for TMD symptoms (90). Similarly, EMG biofeedback has been used to train individuals for relaxation of the masticatory elevator muscles. While EMG biofeedback is an effective procedure for targeted muscle relaxation, its efficacy is improved when combined with other behavioral treatments (32). Behavioral management requires more than simply learning how to decrease motor cortex activation of a specific muscle $(91,92)$. Overall, general relaxation skills or mindfulness training tend to be superior for any chronic pain (93-95) and augment local treatment of behaviors well.

Regardless of which strategy is taken to teach a neutral position of the mandible, the same requirements must be met: patient adherence, clinician persistence with biweekly or monthly follow-up and problem-solving, retraining in the targeted skill towards mastery, and exploring antecedents and barriers. With a goal to transform, for example, overuse behaviors that occur during much of the day to overuse behaviors that seldom occur during the day, the time required for this type of behavioral change varies across individuals: some individuals will describe a nearly
$100 \%$ resolution within a period as short as 2 weeks, while others will require up to a year. Patience and persistence on the part of both patient and clinician is required, but continued investigation into antecedents and co-factors is also required. For example, poorly managed back pain (leading to flareups) or ongoing family stressors, either of which can trigger bouts of masticatory overuse behavior, will hinder or even prevent progress for patients in making desired behavioral changes. Overall body tension or fearavoidance beliefs will similarly impact negatively on the potential for progress. At present, there is no empirical evidence describing this type of tailored care for TMDs. Such approaches have become the standard for back pain and are recommended for TMDs, pending outcomes of necessary clinical trials (96).

\section{Person-level behaviors}

Person-level behavioral factors will contribute to masticatory system non-functional behaviors as well as interfere with local treatments oriented at changing the behaviors. These factors include the following, along with sample effects on behavioral change:

* Depression, which affects motivation;

* Anxiety, which increases general muscle tonus, serves as a proximal trigger for reactive behaviors, and is associated with worry and ruminative thoughts that increase trait-like tendencies for these behaviors;

* Post-traumatic stress disorder, which has effects similar to those of anxiety;

* Other comorbid pain disorders, which act as recurrent triggers for reactive behavior;

- General body tension, which may be regional (neck and masticatory system) or general (whole body), and which increases the difficulty in learning to control masticatory muscle activity;

* Operant behaviors, which reflect secondary gains and can interfere with local behavioral therapies;

* General stress, which increases overall body tonus and general reactivity;

* Poor time-management or assertiveness skills, which contribute to inability to make behavioral change a priority and which increase general stress;

* Absence of relaxation skills, which creates a substantial barrier in learning the local skill of masticatory muscle control;

* Fear-avoidance, which fosters continued muscle response as a guarding behavior; 
* Illness behavior, which affects motivation to change and confidence in one's ability to create that change;

* And pain-related disability, which increases probability of poor adherence to behavioral change.

The above list is of course not exhaustive but does represent prevalent problems that trigger non-functional behaviors or act as barriers to local behavioral treatment for those behaviors. In addition to these factors affecting oral masticatory behaviors, these factors can also affect each other, highlighting the complexity of behavior: a jaw clench event could just be a habitual behavior, or it could be tied into one or many of the person-related behavioral factors stated here. Each of these factors should be actively considered by the clinician at outset of evaluation with regard to magnitude and probable relevance to the TMDs, non-functional behaviors, and pain. This assessment should then be incorporated into the treatment as necessary, depending on overall patient response to initial therapies. In addition, identified patterns of antecedents leading to non-functional behaviors or to pain amplification should be noted. Each of these factors has its own specific treatment needs and approaches, and a detailed and critical description is beyond the scope of this review.

\section{Conclusions}

Behavior associated with mandibular function transcends physical diagnoses, and behavior relevant to TMDs includes functional, non-functional, and person-level types. Non-functional behaviors contribute to TMD onset and persistence, but mechanism(s) for how the behaviors contribute to pain in particular remain unknown. Personlevel behaviors contribute to chronic pain in general but TMD-specific evidence is limited. Assessment and management of these behaviors requires that the clinician utilize principles of behavioral change, address complex patterns due to interaction of multiple behaviors, and continually problem-solve with the patient. Finally, successful behavioral change by patients depends on substantial care and time by the clinician.

\section{Acknowledgments}

Funding: None.

\section{Footnote}

Provenance and Peer Review: This article was commissioned by the Guest Editors (Stephen Feinberg and Louis Mercuri) for the series "Temporomandibular Joint Disorders Diagnosis and Management - What Does the Future Hold?" published in Frontiers of Oral and Maxillofacial Medicine. The article has undergone external peer review.

Conflicts of Interest: Both authors have completed the ICMJE uniform disclosure form (available at https://fomm. amegroups.com/article/view/10.21037/fomm-20-65/coif). The series "Temporomandibular Joint Disorders Diagnosis and Management - What Does the Future Hold?" was commissioned by the editorial office without any funding or sponsorship. The authors have no other conflicts of interest to declare.

Ethical Statement: The authors are accountable for all aspects of the work in ensuring that questions related to the accuracy or integrity of any part of the work are appropriately investigated and resolved.

Open Access Statement: This is an Open Access article distributed in accordance with the Creative Commons Attribution-NonCommercial-NoDerivs 4.0 International License (CC BY-NC-ND 4.0), which permits the noncommercial replication and distribution of the article with the strict proviso that no changes or edits are made and the original work is properly cited (including links to both the formal publication through the relevant DOI and the license). See: https://creativecommons.org/licenses/by-nc-nd/4.0/.

\section{References}

1. Murray GM, Peck CC. Physiology of the Masticatory System. In: Gremillion HA, Klasser GD, editors.

Temporomandibular Disorders: A Translational Approach from Basic Science to Clinical Applicability. Switzerland: Springer International Publishing, 2018:35-64.

2. Shore NA. Occlusal Equilibration and Temporomandibular Joint Dysfunction. Philadelphia: Lippincott, 1959.

3. Moulton RE. Emotional factors in non-organic temporomandibular joint pain. Dent Clin North Am 1966:609-20.

4. Kaplan SEF, Ohrbach R. Self-report of waking-state oral parafunctional behaviors in the natural environment. J Oral Facial Pain Headache 2016;30:107-19.

5. Markiewicz MR, Ohrbach R, McCall WD Jr. Oral Behaviors Checklist: Reliability of Performance in Targeted Waking-state Behaviors. J Orofac Pain 
2006;20:306-16.

6. Barbosa C, Manso MC, Reis T, et al. Cultural equivalence, reliability and utility of the Portuguese version of the Oral Behaviors Checklist. J Oral Rehabil 2018;45:924-31.

7. Raphael KG, Sirois DA, Janal MN, et al. Sleep bruxism and myofascial temporomandibular disorders: a laboratorybased polysomnographic investigation. J Am Dent Assoc 2012;143:1223-31.

8. Conti PCR, Stuginski-Barbosa J, Bonjardim LR, et al. Contingent electrical stimulation inhibits jaw muscle activity during sleep but not pain intensity or masticatory muscle pressure pain threshold in self-reported bruxers: a pilot study. Oral Surg Oral Med Oral Pathol Oral Radiol 2014;117:45-52.

9. Turner JA. Comparison of group progressive-relaxation training and cognitive-behavioral group therapy for chronic low back pain. J Consult Clin Psychol 1982;50:757-65.

10. Fordyce $W$, McMahon R, Rainwater G, et al. Pain complaint-exercise performance relationship in chronic pain. Pain 1981;10:311-21.

11. Fordyce WE. The validity of pain behavior measurement. In: Melzack R, editor. Pain Measurement and Assessment. New York: Raven Press, 1983:145-53.

12. Fordyce WE. Behavioral Methods for Chronic Pain and Illness. Saint Louis: C.V. Mosby Company, 1976.

13. Dworkin SF, LeResche L. Research Diagnostic Criteria for Temporomandibular Disorders: Review, Criteria, Examinations and Specifications, Critique. J Craniomandib Disord 1992;6:301-55.

14. Ohrbach R, Dworkin SF. The Evolution of TMD Diagnosis: Past, Present, Future. J Dent Res 2016;95:1093-101.

15. Wruble MK, Lumley MA, McGlynn FD. Sleeprelated bruxism and sleep variables: a critical review. J Craniomandib Disord 1989;3:152-8.

16. Moss RA, Villarosa GA, Cooley JE, et al. Masticatory muscle activity as a function of parafunctional, active and passive oral behavioural patterns. J Oral Rehabil 1987;14:361-70.

17. Clark GT, Beemsterboer PL, Solberg WK, et al. Nocturnal electromyographic evaluation of myofascial pain dysfunction in patients undergoing occlusal splint therapy. J Am Dent Assoc 1979;99:607-11.

18. Westling L, Carlsson GE, Helkimo M. Background factors in craniomandibular disorders with special reference to general joint hypermobility, parafunction, and trauma. J Craniomandib Disord 1990;4:89-98.
19. Nilner M. Relationships between oral parafunctions and functional disturbances and diseases of the stomatognathic system among children aged 7-14 years. Acta Odontol Scand 1983;41:167-72.

20. Look JO, Schiffman EL, Truelove EL, et al. Reliability and validity of Axis I of the Research Diagnostic Criteria for Temporomandibular Disorders (RDC/TMD) with proposed revisions. J Oral Rehabil 2010;37:744-59.

21. Ohrbach R. Assessment and further development of RDC/TMD Axis II biobehavioural instruments: a research programme progress report. J Oral Rehabil 2010;37:784-98.

22. Moseley GL, Hodges PW. Reduced variability of postural strategy prevents normalization of motor changes induced by back pain: a risk factor for chronic trouble? Behav Neurosci 2006;120:474-6.

23. Moseley GL, Nicholas MK, Hodges PW. A randomized controlled trial of intensive neurophysiology education in chronic low back pain. Clin J Pain 2004;20:324-30.

24. Moore JE, Von Korff M, Cherkin D, et al. A randomized trial of a cognitive-behavioral program for enhancing back pain self care in a primary care setting. Pain 2000;88:145-53.

25. Linton SJ. A review of psychological risk factors in back and neck pain. Spine 2000;25:1148-56.

26. Asmundson GJG, Norton GR, Allerdings MD. Fear and avoidance in dysfunctional chronic back pain patients'. Pain 1997;69:231-6.

27. Vlaeyen JWS, Kole-Snijders AMJ, Boeren RGB, et al. Fear of movement/(re)injury in chronic low back pain and its relation to behavioral performance. Pain 1995;62:363-72.

28. Spinhoven P, Linssen ACG. Behavioral treatment of chronic low back pain. I. Relation of coping strategy use to outcome. Pain 1991;45:29-34.

29. Keefe FJ, Bradley LA, Crisson JE. Behavioral assessment of low back pain: identification of pain behavior subgroups. Pain 1990;40:153-60.

30. Waddell G, Pilowsky I, Bond MR. Clinical assessment and interpretation of abnormal illness behavior in low back pain. Pain 1989;39:41-53.

31. Crider A, Glaros AG, Gevirtz RN. Efficacy of biofeedback-based treatments for temporomandibular disorders. Applied Psychophysiology and Biofeedback 2005;30:333-45.

32. Mishra KD, Gatchel RJ, Gardea MA. The relative efficacy of three cognitive-behavioral treatment approaches to temporomandibular disorders. J Behav Med 2000;23:293-309. 
33. Glaros AG. Awareness of physiological responding under stress and nonstress conditions in temporomandibular disorders. Biofeedback Self Regul1996;21:261-72.

34. Rudy TE, Turk DC, Kubinski JA, et al. Differential treatment responses of TMD patients as a function of psychological characteristics. Pain 1995;61:103-12.

35. Kino K, Sugisaki M, Haketa T, et al. The comparison between pains, difficulties in function, and associating factors of patients in subtypes of temporomandibular disorders. J Oral Rehabil 2005;32:315-25.

36. Rantala MA, Ahlberg J, Suvinen TI, et al. Chronic myofascial pain, disk displacement with reduction and psychosocial factors in Finnish non-patients. Acta Odontol Scand 2004;62:293-7.

37. Kornaat PR, Bloem JL, Ceulemans RY, et al. Osteoarthritis of the knee: association between clinical features and MR imaging findings. Radiology 2006;239:811-7.

38. McAlindon TE, Cooper C, Kirwan JR, et al. Determinants of disability in osteoarthritis of the knee. Ann Rheum Dis 1993;52:258-62.

39. Castroflorio T, Bargellini A, Rossini G, et al. Sleep bruxism and related risk factors in adults: a systematic literature review. Arch Oral Biol 2017;83:25-32.

40. Manfredini D, Ahlberg J, Winocur E, et al. Management of sleep bruxism in adults: a qualitative systematic literature review. J Oral Rehabil 2015;42:862-74.

41. Al-Jewair T, Shibeika D, Ohrbach R. Temporomandibular disorders and their association with sleep disorders in adults: a systematic review. J Oral Facial Pain Headache 2021;35:41-53.

42. Hirsch JA, McCall WD, Jr., Bishop B. Jaw dysfunction in viola and violin players. J Am Dent Assoc 1982;104:838-43.

43. Glaros AG, Marszalek JM, Williams KB. Longitudinal multilevel modeling of facial pain, muscle tension, and stress. J Dent Res 2016;95:416-22.

44. Ohrbach R, Michelotti A. The role of stress in the etiology of oral parafunction and myofascial pain. In: Laskin D, Renapurkar SK, editors. Current Controversies in the Management of Temporomandibular Disorders: Oral and Maxillofacial Surgery Clinics of North America. Philadelphia, PA: Elsevier, 2018:369-80.

45. Glaros AG, Hanson AH, Ryen CC. Headache and oral parafunctional behaviors. Appl Psychophysiol Biofeedback 2014;39:59-66.

46. Ohrbach R, Bair E, Fillingim RB, et al. Clinical orofacial characteristics associated with risk of first-onset TMD: the OPPERA prospective cohort study. J Pain 2013;14:T33-T50.
47. Michelotti A, Cioffi I, Festa P, et al. Oral parafunctions as risk factors for diagnostic TMD subgroups. J Oral Rehabil 2010;37:157-62.

48. van Selms MK, Lobbezoo F, Visscher CM, et al. Myofascial temporomandibular disorder pain, parafunctions and psychological stress. J Oral Rehabil 2008;35:45-52.

49. Chen C-Y, Palla S, Emi S, et al. Nonfunctional tooth contact in healthy controls and patients with myogenous facial pain. J Orofac Pain 2007;21:185-93.

50. Ohrbach R, Fillingim RB, Mulkey F, et al. Clinical findings and pain symptoms as potential risk factors for chronic TMD: Descriptive data and empirically identified domains from the OPPERA case-control study. J Pain 2011;12:T27-T45.

51. Fragoso YD, Alves HH, Garcia SO, et al. Prevalence of parafunctional habits and temporomandibular dysfunction symptoms in patients attending a tertiary headache clinic. Arq Neuropsiquiatr 2010;68:377-80.

52. Winocur E, Gavish A, Finkelshtein T, et al. Oral habits among adolescent girls and their association with symptoms of temporomandibular disorders. J Oral Rehabil 2001;28:624-9.

53. Glaros AG, Williams K, Lausten L. Diurnal variation in pain reports in temporomandibular disorder patients and control subjects. J Orofac Pain 2008;22:115-21.

54. Pullinger AG, Seligman DA, Solberg WK. Temporomandibular disorders. Part II: Occlusal factors associated with temporomandibular joint tenderness and dysfunction. J Prosthet Dent 1988;59:363-7.

55. Minami I, Akhter R, Albersen I, et al. Masseter motor unit recruitment is altered in experimental jaw muscle pain. J Dent Res 2013;92:143-8.

56. Slade GD, Bair E, By K, et al. Study methods, recruitment, sociodemographic findings, and demographic representativeness in the OPPERA study. J Pain 2011;12:T12-T26.

57. Ohrbach R, Fernández-de-las-Peñas C. Pain Psychology, Behavior, and the Body. In: Fernández-de-las-Peñas C, Mesa-Jiménez J, editors. Temporomandibular Disorders: Man Ther, Exercise and Needling Therapies. Edinburgh: HandSpring Publishing, 2018:291-8.

58. Ohrbach R, Slade GD, Bair E, et al. Premorbid and concurrent predictors of TMD onset and persistence. Eur J Pain 2020;24:145-58.

59. Moseley GL. A pain neuromatrix approach to patients with chronic pain. Man Ther 2003;8:130-40.

60. Litt MD, Porto FB. Determinants of Pain Treatment Response and Nonresponse: Identification of TMD 
Patient Subgroups. J Pain 2013;14:1502-13.

61. Orlando B, Manfredini D, Salvetti G, et al. Evaluation of the effectiveness of biobehavioral therapy in the treatment of temporomandibular disorders: a literature review. Behav Med 2007;33:101-18.

62. Ohrbach R. Biobehavioral Therapy. In: Laskin DM, Greene CS, Hylander WL, editors. TMDs: An EvidenceBased Approach to Diagnosis and Treatment. Hanover Park, IL: Quintessence Publishing Co, Inc., 2006:391-403.

63. Durham J, Al-Baghdadi M, Baad-Hansen L, et al. Selfmanagement programmes in temporomandibular disorders: results from an international Delphi process. J Oral Rehabil 2016;43:929-36.

64. Keefe FJ, Main CJ, George SZ. Advancing psychologically informed practice for patients with persistent musculoskeletal pain: promise, pitfalls, and solutions. Phys Ther 2018;98:398-407.

65. Main CJ, George SZ. Psychologically informed practice for management of low back pain: future directions in practice and research. Phys Ther 2011;91:820-4.

66. Johnson MH, Kazantzis N. Cognitive behavioral therapy for chronic pain: Strategies for the successful use of homework assignments. J Ration Emot Cogn Behav Ther 2004;22:189-218.

67. Feuerstein M, Beattie P. Biobehavioral factors affecting pain and disability in low back pain: mechanisms and assessment. Phys Ther 1995;75:267-80.

68. Gavish A, Winocur E, Menashe S, et al. Experimental chewing in myofascial pain patients. J Orofac Pain 2002;16:22-8.

69. Isberg-Holm AM, Westesson PL. Movement of disc and condyle in temporomandibular joints with clicking: an arthrographic and cineradiographic study on autopsy specimens. Acta Odontol Scand 1982;40:151-64.

70. Boersma K, Linton SJ. How does persistent pain develop? An analysis of the relationship between psychological variables, pain and function across stages of chronicity. Behav Res Ther 2005;43:1495-507.

71. Vlaeyen JW, Linton SJ. Fear-avoidance and its consequences in chronic musculoskeletal pain: a state of the art. Pain 2000;85:317-32.

72. Ohrbach R, Foigelman-Holland D. Contracture. Clinical Cases in Orofacial Pain. Oxford, UK: John Wiley \& Sons, 2017:134-40.

73. Hides JA, Donelson R, Lee D, et al. Convergence and divergence of exercise-based approaches that incorporate motor control for the management of low back pain. J Orthop Sports Phys Ther 2019;49:437-52.
74. Proske U, Gandevia SC. The proprioceptive senses: their roles in signaling body shape, body position and movement, and muscle force. Physiol Rev 2012;92:1651-97.

75. Tsay A, Allen TJ, Proske U, et al. Sensing the body in chronic pain: A review of psychophysical studies implicating altered body representation. Neurosci Biobehav Rev 2015;52:221-32.

76. Jones LE, O'Shaughnessy DF. The pain and movement reasoning model: introduction to a simple tool for integrated pain assessment. Man Ther 2014;19:270-6.

77. Helkimo M. Studies on function and dysfunction of the masticatory system. Acta Odontol Scand 1974;32:255-67.

78. Schiffman EL, Fricton JR, Haley D. The relationship of occlusion, parafunctional habits and recent life events to mandibular dysfunction in a non-patient population. J Oral Rehabil 1992;19:201-23.

79. Glaros AG, Williams K, Lausten L, et al. Tooth contact in patients with temporomandibular disorders. Cranio 2005;23:188-93.

80. Gallo LM, Gross SS, Palla S. Nocturnal masseter EMG activity of healthy subjects in a natural environment. J Dent Res 1999;78:1436-44.

81. Baba K, Clark GT, Watanabe T, et al. Bruxism force detection by a piezoelectric film-based recording device in sleeping humans. J Orofac Pain 2003;17:58-64.

82. Schiffman E, Ohrbach R, Truelove E, et al. Diagnostic Criteria for Temporomandibular Disorders (DC/TMD) for Clinical and Research Applications: Recommendations of the International RDC/TMD Consortium Network and Orofacial Pain Special Interest Group. J Oral Facial Pain Headache 2014;28:6-27.

83. Ohrbach R, Markiewicz MR, McCall WD Jr. Wakingstate oral parafunctional behaviors: specificity and validity as assessed by electromyography. Eur J Oral Sci 2008;116:438-44.

84. Carlson CR, Sherman JJ, Studts JL, et al. The effects of tongue position on mandibular muscle activity. J Orofac Pain 1997;11:291-7.

85. Rocabado M, Iglarsh ZA. The Musculoskeletal Approach to Maxillofacial Pain. Philadelphia, PA: Lippincott Williams and Wilkins, 1991.

86. Spence SH, Sharpe L, Newton-John T, et al. Effect of EMG biofeedback compared to applied relaxation training with chronic, upper extremity cumulative trauma disorders. Pain 1995;63:199-206.

87. Sime WE, DeGood DE. Effect of EMG biofeedback and progressive muscle relaxation trainingon awareness of frontalis muscle tension. Psychophysiology 1977;14:522-30. 
88. Story WP, Durham J, Al-Baghdadi M, et al. Selfmanagement in temporomandibular disorders: a systematic review of behavioural components. J Oral Rehabil 2016;43:759-70.

89. Michelotti A, de Wijer A, Steenks M, et al. Homeexercise regimes for the management of nonspecific temporomandibular disorders. J Oral Rehabil 2005;32:779-85.

90. Riley JL, Myers CD, Currie TP, et al. Self-care behaviors associated with myofascial temporomandibular disorder pain. J Orofac Pain 2007;21:194-202.

91. Miller L. Biofeedback and behavioral medicine: treating the symptom, the syndrome, or the person? Psychotherapy 1994;31:161-9.

92. Flor H, Birbaumer N. Comparison of the efficacy of electromyographic biofeedback, cognitive-behavioral therapy, and conservative medical interventions in the

doi: 10.21037/fomm-20-65

Cite this article as: Ohrbach R, Sharma S. Behavioral therapy for temporomandibular disorders. Front Oral Maxillofac Med 2021;3:37. treatment of chronic musculoskeletal pain. J Consult Clin Psychol 1993;61:653-8.

93. NIH. NIH Technology Assessment Conference on Integration of Behavioraland Relaxation Approaches into the Treatment of Chronic Pain and Insomnia. Bethesda, MD: National Institutes of Health, 1995.

94. Zeidan F, Baumgartner JN, Coghill RC. The neural mechanisms of mindfulness-based pain relief: a functional magnetic resonance imaging-based review and primer. Pain Rep 2019;4:e759.

95. Cherkin DC, Sherman KJ, Balderson BH, et al. Effect of mindfulness-based stress reduction vs cognitive behavioral therapy or usual care on back pain and functional limitations in adults with chronic low back pain: A randomized clinical trial. JAMA 2016;315:1240-9.

96. Temporomandibular Disorders: Priorities for Research and Care. Washington, DC: National Academies Press, 2020. 